\section{Clinical heterogeneity in a family with DKC1 mutation, dyskeratosis congenita and Hoyeraal-Hreidarsson syndrome in first cousins}

\author{
Cristina Olivieri, ${ }^{1}$ Anna Mondino, ${ }^{1}$ \\ Matteo Chinello, ${ }^{2}$ Alessandra Risso, ${ }^{3}$ \\ Enrico Finale, ${ }^{4}$ Marina Lanciotti, 5 \\ Andrea Guala 4
}

1Department of Public and Pediatric Health Sciences, University of Turin; 2Department of Pediatric Hematology and Oncology, Policlinico G.B. Rossi, Verona; ${ }^{3}$ Departement of Hematology, City of Health and Science, University of Turin; 4Unit of Pediatrics, Castelli Hospital, Verbania; 5Department of Pediatric Hematology Oncology and Bone Marrow Transplant, G. Gaslini Children's Hospital, Genoa, Italy

\begin{abstract}
Dyskeratosis congenita (DC) is an inherited bone marrow failure disorder characterized by mucocutaneous features (skin pigmentation, nail dystrophy and oral leukoplakia), pulmonary fibrosis, hematologic and solid malignancies. Its severe form, recognized as Hoyeraal-Hreidarsson syndrome (HHS), also includes cerebellar hypoplasia, microcephaly, developmental delay and prenatal growth retardation. In literature phenotypic variability among DC patients sharing the same mutation is wellknown. To our knowledge this report describes for the first time a family of DC patients, characterized by a member with features of classic DC and another one with some features of HHS, both with the same mutation in $D K C 1$. Our family confirms again that one mutation can be associated with different phenotypes and different hematological manifestations. It's possible to speculate that there are likely to be patients who do not clinically fit neatly into either classical DC or HHS, but whose clinical features are due to mutations in $\mathrm{DKCl}$ or in genes responsible for autosomal DC/HHS
\end{abstract}

\section{Introduction}

Dyskeratosis Congenita (DC) was first described in 1906 by Zinsser. ${ }^{1}$ Many genes involved in telomere length maintenance have been reported as causive in patients with DC.2,3 Telomers are implicated in cel- lular aging and tumorigenesis. Defects in telomere biology related to DC can cause bone marrow failure (BMF) and malignancies.

The classical diagnostic triad described in DC patients includes reticular pigmentation of the skin, nail dystrophy and mucosal leukoplakia. BMF develops during adolescence and it is one of the major causes of death, together with immunodeficiency, pulmonary complications, and malignancies.

Three inheritance patterns have been described in DC: X-linked recessive (the most frequent, due to DKC1 mutations), autosomal dominant and autosomal recessive.

As widely reported in literature, DC is characterized by wide clinical heterogeneity. 4,5

Different forms of DC are reported. 6 Patients with classic DC present two or more of the diagnostic mucocutaneous features or one of these features together with BMF. Patients with atypical DC show isolated $\mathrm{BMF}$, or pulmonary fibrosis only or less common clinical manifestations such as alopecia, hyperhidrosis, ungual atrophy and periodontal disease. ${ }^{7}$ Patients with silent $D C$ show no clinical manifestation, but they carry a pathogenic mutation. Severe variants of DC are Hoyeraal-Hreidarsson (HHS) and Revesz syndromes. The typical features of HHS are cerebellar hypoplasia, associated with microcephaly, immunodeficiency, progressive BMF, intrauterine growth retardation (IUGR), developmental delay. 2,8 Revesz syndrome is characterized by the presence of bilateral exudative retinopathy, intracranial calcifications and IUGR; also additional features of DC may be present. 9

Many of the mutated genes in classic DC are also involved in HHS. ${ }^{2}$ In particular many families with DC or with HHS are described in literature. ${ }^{10-12}$

In this report we describe two cousins with the same mutation in $\mathrm{DKC1}$ : one with classic DC and the other one with some features of HHS.

\section{Case Report}

A ten-month-old child (proband) was admitted to our department for persistent fever and petechial rash in the genital area. The patient appeared in good health condition. The petechial rash spread over neck and shoulders. Cardiac, respiratory and abdominal examination was normal. Irregular scars were present on the oral mucosa, resulting from the previous surgi-
Correspondence: Enrico Finale, Pediatria, Ospedale Castelli Verbania, Via Fiume n.18, 28922 Verbania (VB), Italy.

Tel.: +39.3930128648

E-mail: enrico.finale@gmail.com

Key words: Dyskeratosis Congenital, HoyeraalHreidarsson Syndrome, gene mutation, phenotypic variability.

Contributions: the authors contributed equally.

Conflict of interest: the authors declare no potential conflict of interest.

Received for publication: 4 July 2017.

Revision received: 7 August 2017.

Accepted for publication: 7 August 2017.

This work is licensed under a Creative Commons Attribution NonCommercial 4.0 License (CC BY-NC 4.0).

C Copyright C. Olivieri et al., 2017

Licensee PAGEPress, Italy

Pediatric Reports 2017; 9:7301

doi:10.4081/pr.2017.7301

cal removal of a leukoplakia (Figure 1). Medical history revealed IUGR started at the 32 nd week of gestation. The child was born by caesarean section at 35 weeks of gestation. Nothing relevant was reported in his family history.

The cell blood count revealed anemia, thrombocytopenia and neutropenia. A bone marrow aspiration was performed to rule out the presence of blasts, signs of maturity dysplasia or hemophagocytosis and no atypical cells were revealed, but a trilinear bone marrow hypocellularity was shown. Culture assays showed a decreased number of progenitor cells (CFU-GM and BFU-E). Parvovirus B19, Epstein Barr Virus and Cytomegalovirus infections were excluded by serological tests. Cytogenetic analysis showed a normal 46 XY karyotype. Fanconi Anemia (FA) was ruled out by a standard chromosomal breakage test with diepoxybutane and mitomycin $\mathrm{C}$, that did not reveal any chromosomal hypersensitivity to these clastogenic agents. Also cell cycle analysis was in the normal range. Molecular analysis of PRF1, MUNC 13-18 and STX genes were performed to exclude a familial hemophagocytic lymphoistiocitosis (FHL) and no sequence alteration was revealed.

The presence of the oral mucosal leukoplakia led us to consider an immunodeficiency disorder. Immunoglobulin blood values were normal, while the immunophenotyping of blood lymphocytes identified a reduction of $\mathrm{B}$ lymphocytes $(\mathrm{CD} 19+=$ 
63/uL) and an increase in CD8 + lymphocytes, in particular with reference to the effector component.

The patient was treated with red blood cell and immunoglobulin infusions with a considerable clinical improvement. He was discharged with a diagnosis of post-infectious cytopenia. Four weeks later, he came back to the hospital because of nocturnal fever.

Thrombocytopenia and neutropenia were still present (platelets: 57,000/uL, neutrophils: $800 / \mathrm{uL}$ ). This result, coupled with the leukoplakia, led us to consider the diagnosis of DC. Telomere length resulted extremely short if related to the patient's age (Figure 2). Genes coding for $D K C 1$, TERT e TERC were analyzed and a mutation in $D K C 1$ gene $(1156 \mathrm{G}>\mathrm{A})$ was detected.

Since $D K C 1$ gene is on chromosome $\mathrm{X}$, a familiar survey was performed in the maternal line and a three-year-old first cousin with cerebellar hypoplasia, micro-

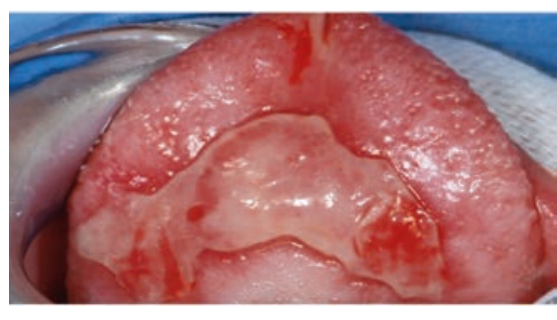

Figure 1. Tongue leukoplachia. cephaly and delay in walking was identified as a mutation carrier. He showed characteristics typical of DC, such as significant nail dystrophy and reticulated skin pigmentation and some features typical of HHS. Unlike the proband, blood cell counts and lymphocyte immunophenotype were normal although he presented the same mutation in the DKC1 gene (1156 G>A). The mothers of patients, who are sisters, were asymptomatic carriers of the same DKC1 mutation.

\section{Discussion}

In this report, for the first time, we describe two consanguineous patients with the same mutations in DKC1 showing different phenotype.

The proband presents features of classic $D C$ : oral mucosal leukoplakia (one of the typical mucocutaneous manifestations) and BMF with immunodeficiency.

Indeed, classic DC is characterized by typical mucocutaneous manifestations including abnormal skin pigmentation, arranged in a reticular pattern, and poikilodermatous alterations consisting in atrophy or telangiectasia. Progressive nail dystrophy is reported in about $90 \%$ of patients. Mucosal changes, such as leukoplakia involving buccal mucosa, tongue and oropharynx, are described. Furthermore patients with DC frequently develop BMF. At onset, bone marrow is usually normal, but hematopoietic cells are gradually replaced by fibrotic tissue, with a consequent development of pancytopenia and increase of the mortality risk for secondary

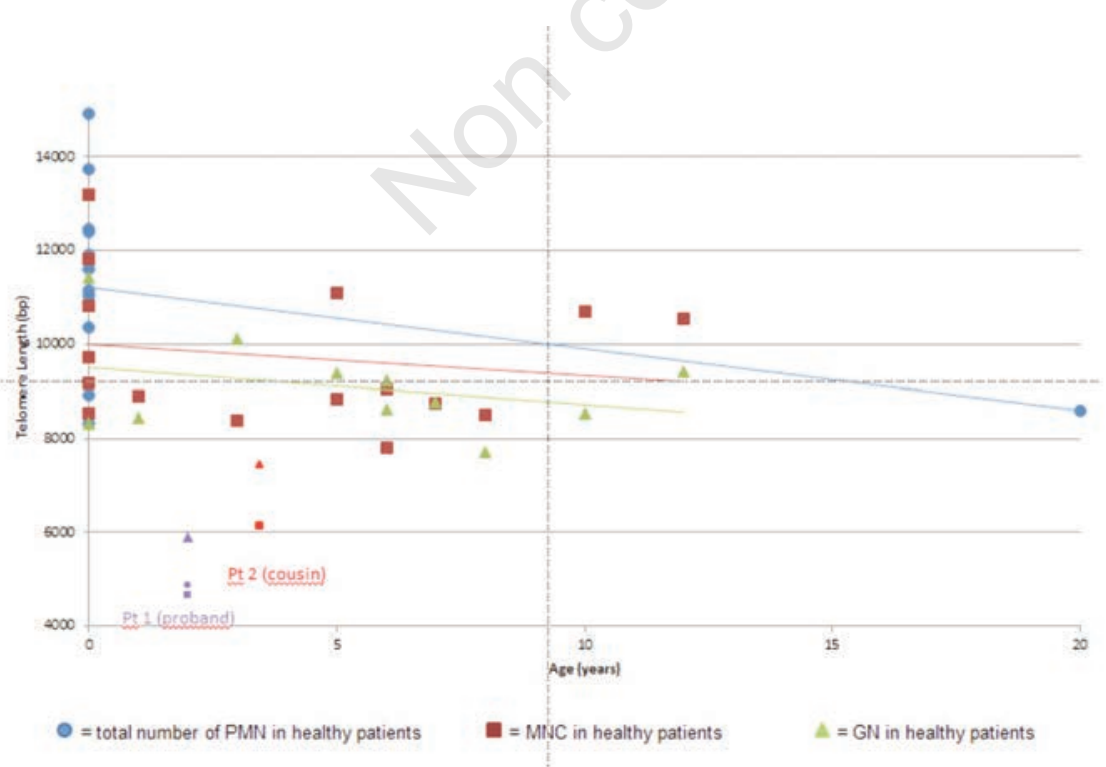

Figure 2. Telomere length in the patients (proband and cousin) and controls (healthy patients). Pt= patient; $\mathrm{PMN}=$ Polymorphonuclear leucocytes; $\mathrm{MNC}=$ Mononuclear cells; GN= Neutrophil granulocytes. infections. ${ }^{13,14}$

The proband's cousin presents abnormalities typical of classic $D C$, such as significant nail dystrophy and reticulated skin pigmentation. He also shows some features typical of HHS, such as cerebellar hypoplasia and microcephaly, but not immunodeficiency nor BMF. Patients affected by HHS show very short telomeres, as described in DC patients. The syndrome is typically characterized by cerebellar hypoplasia, which is mandatory for diagnosis of HHS, immunodeficiency and BMF.9,15 It is often associated with IUGR, microcephaly and developmental delay. In addition to these typical HHS features, these patients often show many characteristics of classic DC. ${ }^{2}$

Despite their different phenotypes, the two cousins share the same mutation in exon 12 of the dyskerin gene ( $D K C l$ gene $1156 \mathrm{G}>\mathrm{A})$. This gene encodes for ubiquitously expressed dyskerin, a component of the human telomerase complex. ${ }^{16}$ Many mutation in DKC1 have been previously observed both in patients with DC and in patient with HHS, in a X-linked recessive transmission. 2,12,17

In particular the mutation identified in our probands (1156 $\mathrm{G}>\mathrm{A}$ in exon 12) is rare $^{18}$ and it has already been described in DC patients and HHS patients. ${ }^{2}$

Vulliamy's article ${ }^{12}$ reports a family whose index case presents this latter mutation and clinical features of both classic DC and HHS.

\section{Conclusions}

Our family confirms again that same mutation can be associated with different phenotypes and different hematological manifestations, while different phenotypes in the same family further confirms the high variability of the disease expressivity and penetrance. It's possible to speculate that there are likely to be patients who do not clinically fit neatly into either classical DC or HHS, but whose clinical features are due to mutations in $\mathrm{DKCl}$ or in genes responsible for autosomal DC/HHS.

\section{References}

1. Zinsser F. Atrophia cutis reticularis cum pigmentione, dystrophia unguium et leukokeratosis oris. Ikonogr Dermatol 1906;5:219-23.

2. Glousker G, Touzot F, Revy P, et al. Unraveling the pathogenesis of Hoyeraal-Hreidarsson syndrome, a complex telomere biology disorder. Br J Haematol 2015;170:457-71. 
3. Yamaguchi H, Sakaguchi H, Yoshida K, et al. Clinical and genetic features of dyskeratosis congenita, cryptic dyskeratosis congenita, and HoyeraalHreidarsson syndrome in Japan. Int J Hematol 2015;102:544-52.

4. Kirwan M, Dokal I. Dyskeratosis congenita: a genetic disorder of many faces. Clin Genet 2008;73:103-12.

5. Vulliamy TJ, Dokal I. Dyskeratosis congenita: the diverse clinical presentation of mutations in the telomerase complex. Biochimie 2008;90:122-30.

6. Bessler M, Wilson DB, Mason PJ. Dyskeratosis congenita. FEBS Lett 2010;584:3831-8.

7. Dokal I. Dyskeratosis congenita in all its forms. Br J Haematol 2000;110:76879 .

8. Aalfs CM, Van den Berg H, Barth PG, et al. The Hoyeraal-Hreidarsson syndrome: the fourth case of a separate entity with prenatal growth retardation, progressive pancytopenia and cerebel- lar hypoplasia. Eur J Pediatr 1995; 154:304-8

9. Savage SA, Alter BP. Dyskeratosis congenita. Hematol Oncol Clin North Am 2009;23:215-31.

10. Coelho JD, Lestre S, Kay T, et al. Dyskeratosis congenita: two siblings with a new missense mutation in the DKC1 gene. Pediatr Dermatol 2011;28:464-6.

11. Lai W, Deng WP, Liu X, et al. A recurrent p. A353V mutation in $\mathrm{DKC1}$ responsible for different phenotypes of dyskeratosis congenita in a Chinese family. J Dermatol Sci 2011;63:122-4.

12. Vulliamy TJ, Marrone A, Knight SW, et al. Mutations in dyskeratosis congenita: their impact on telomere length and the diversity of clinical presentation. Blood 2006;107:2680-5.

13. Baran I, Nalcaci R, Kocak M. Dyskeratosis congenita: clinical report and review of the literature. Int J Dent Hyg 2010;8:68-74.
14. Auluck A. Dyskeratosis congenita. Report of a case with literature review. Med Oral Patol Oral Cir Bucal 2007;12:E369-73.

15. Savage SA, Bertuch AA. The genetics and clinical manifestations of telomere biology disorders. Genet Med 2010;12: 753-64.

16. Vulliamy T, Dokal I. Dyskeratosis congenita. Semin Hematol 2006;43:15766.

17. Pearson T, Curtis F, Al-Eyadhy A, et al. An intronic mutation in DKC1 in an infant with Høyeraal-Hreidarsson syndrome. Am J Med Genet A 2008; 146A:2159-61.

18. Zeng XL, Thumati NR, Fleisig HB, et al. The accumulation and not the specific activity of telomerase ribonucleoprotein determines telomere maintenance deficiency in X-linked dyskeratosis congenita. Hum Mol Genet 2012;21: 721-9. 